\title{
La desorientación de la edad propia: una forma de síndrome de falsa identificación delirante o de deterioro intelectual
}

\author{
Omar Al-Gommer, MRCPsych, Specialist \\ Registrar \\ Hamdy Moselhy, MRCPsych, Consultant \\ Psychiatrist \\ UNITED KINGDOM
}

\begin{abstract}
RESUMEN - Se presentan dos casos de pacientes con esquizofrenia y que habían infravalorado en 10 años su edad, que acuden por primera vez a consulta psiquiátrica. Uno de los pacientes presentados no toma neurolépticos y no está institucionalizado. También se presenta una revisión sobre la desorientación de la edad propia.
\end{abstract}

\section{Introducción}

A menudo se ha observado que pacientes con estados amnésicos son proclives a estimar a la baja sus edades. En los casos de síndrome de Korsakov, ésta estimación a la baja es muy evidente y su extensión parece estar determinada, al menos en parte, por la duración de la amnesia retrógrada. También puede darse una estimación de la edad a la baja, menos extrema, en las fases tempranas de la recuperación tras una lesión cerebral (Zangwill y Cautab 1959).

Otros estudios (Crow y Mitchell 1978, Steven et al. 1978, Smith y Oswald 1976) concluyeron que aproximadamente un $25 \%$ de la población de pacientes esquizofrénicos crónicos ingresados estimaban su edad incorrectamente, con un margen de error de cinco años o más. Crow y Mitchell (1975) realizaron un estudio sobre la edad subjetiva de 237 pacientes varones crónicos ingresados, diagnosticados de esquizofrenia, en el que un $25 \%$ creían ser 5 ó más años más jóvenes de lo que eran en realidad. La media de edad verdadera del grupo con desorientación en la edad era de 59 años y la media de la duración del internamiento era de 26 años. Estos datos fueron confirmados por otro estudio reciente (Manschrech et al. 2000) en el que un $30 \%$ de los pacientes esquizofrénicos, ingresados en una institu- 
ción para enfermos crónicos, estaban desorientados con respecto a su edad.

Los resultados de Smith y Oswald (1976) mostraban que la media de edad de su muestra era de 53 años y la media de duración del internamiento era de 18 años. Selten y Cath (1994) estudiaron la prevalencia de la desorientación en la edad en pacientes esquizofrénicos en residencias de larga estancia alemanas. Encontraron que la media de edad era de 47,5 años, la media de duración del último ingreso era de 13 años y tan sólo un paciente de los 55 hombres y las 32 mujeres creía ser 5 más joven de lo que era. Los autores achacaron estos hallazgos divergentes a la baja estimulación en los hospitales británicos y a la variación regional en la gravedad de la esquizofrenia.

La desorientación de la edad se asocia con la desorientación temporal, en la que los pacientes sistemáticamente infra-estiman el año en el que se encuentran y la duración de su estancia en el hospital.

Para algunos, aunque no para todos los individuos, los errores en la información temporal son coherentes con el concepto de su propia edad. Liddle y Crow (1984) consideran que la desorientación de la edad podría ser un marcador de una forma de esquizofrenia crónica, caracterizada por un grave y amplio deterioro del intelecto, y que posiblemente pudiera ser una secuela de un proceso patológico activo desde la infancia temprana.

Presentamos dos casos de pacientes con diagnóstico de esquizofrenia que estaban desorientados con respecto a su edad. Estos pacientes no infra-estimaban el paso del tiempo. En el Caso 1 se trataba de un delirio aislado, pero en el Caso 2 podía ser parte de un deterioro cognoscitivo. Nosotros sugerimos que, en caso de delirio, lo más probable es que forme parte de un síndrome de falsa identificación delirante. En el síndrome de falsa identificación delirante es típico que se involucre la falsa identificación de personas, pero también pueden intervenir la falsa identificación de lugares, de objetos o incluso de partes del cuerpo (Moselhy y Oyebode 1997).

\section{Caso 1}

El Sr. A es un hombre de 41 años trasladado desde un Hospital General de Distrito después de ser evaluado por un psiquiatra consultor cuando se implantó la Ley de Salud Mental de 1983. Cuando fue admitido en el Hospital General, sufría ulceraciones en las piernas, infección y anemia graves debidos al propio abandono. Fue encontrado por la policía en estado de grave abandono cuando merodeaba por el centro de la ciudad. Contó que llevaba 4 meses con los problemas de sus piernas pero "el ordenador" le había impedido ir al hospital.

Describía toda su situación como si estuviese controlado por algunos ordenadores que tenían bajo control su pensamiento, organizaban sus movimientos y hablaban a través de su voz. Decía que llevaba teniendo esas sensaciones desde hacía 3 meses. Reconocía ser un "sin techo", sufría una grave pérdida de peso y a veces observaba que murmuraba consigo mismo. Declaró que había perdido su trabajo en una fábrica de coches y que le quitaron la casa porque el ordenador de la universidad hizo que ocurrieran ambas cosas.

Al contactar con el agente de la propiedad cuyo nombre había mencionado, se confirmó el embargo de la casa del Sr. A, pero había tenido lugar al menos 3 años antes. El agente informó que después de esto, el Sr. A parece se negó a aceptar que la casa ya no le pertenecía y acampó en el 
cobertizo. Al parecer, los propietarios se quejaron y el agente de la propiedad se ocupó de que el Sr. A fuera advertido y desalojado. Desde entonces, el agente de la propiedad dijo que le había visto regularmente caminando calle arriba y abajo como un "vagabundo".

El paciente dijo que en una etapa de su vida había estado casado durante 9 años, pero se divorció. También dijo que no tenía contacto con sus hermanos o padres y no tenía ni idea de dónde vivían ni sabía cómo obtener información adicional. Aunque dijo que llevaba trabajando desde 4 meses antes de su ingreso, no pudimos encontrar su historial en el lugar de trabajo señalado. Dejó la escuela a los 15 años sin graduarse y tuvo numerosos empleos, el último durante 2 años como operador de maquinaria en una fábrica de coches. Contó que le gustaba pintar al óleo, leer novelas y la jardinería.

Negaba cualquier historia de abuso de alcohol o drogas. Negaba su ingreso anterior en ningún hospital psiquiátrico y su médico de cabecera informó de que este paciente fue visto por última vez en su consulta hacía 5 años y que su historia no mostraba ningún antecedente médico o psiquiátrico que pudiera arrojar luz sobre la situación del paciente.

Durante la entrevista se mostraba poco preciso, desaliñado, sin afeitar, poco aseado, con la expresión facial invariable y sin contacto ocular. No hablaba, salvo cuando le hablaban. Su humor era aplanado y negaba haber sufrido alucinaciones. No podía recordar cómo o por qué había acabado en el hospital. Podía señalar correctamente su año de nacimiento cuando se le preguntaba y no tenía dudas acerca de en qué año estaba; aún así mantenía invariablemente que su edad era de 31 años; este error resistía cualquier intento de corrección. En Año Nuevo, declaró que su año de nacimiento era un año después que lo dicho anteriormente y mantuvo que su edad era de 31 años, aunque decía cuál era el nuevo año correctamente.

Actualmente no tiene conciencia de su enfermedad mental y cree que está en el hospital y tomando medicación debido a sus ulceraciones en las piernas (incluso después de que sanaran) y solicita ayuda para buscar alojamiento. Su fecha de nacimiento se verificó a través del Registro de Médicos Generalistas y de su número de la Seguridad Social. Permaneció en un hospital general durante 2 meses para tratar las ulceraciones de sus piernas, su anemia grave y su grave pérdida de peso.

La revisión efectuada mostró que su nivel de hemoglobina era de 4,2 mg./l. y necesitó transfusiones de sangre en tres ocasiones, hasta alcanzar los $12 \mathrm{mg}$./l. antes de su derivación al hospital psiquiátrico. No se hallaron enfermedades metabólicas en las analíticas rutinarias y la ecografía abdominal que se le practicó fue normal. Aunque su RM fue normal, el EEG mostró una onda lenta inespecífica en la región anterior y temporal medial izquierdas, que sugieren una atrofia moderada. Su medicación fue haloperidol (10 mg./día, en 2 tomas) durante 3 meses, que se sustituyó posteriormente por trifluoperazina (10 mg./ día, en 3 tomas).

Durante los 6 meses que estuvo bajo nuestra observación se mostró muy aislado, exhibiendo sintomatología negativa, permaneciendo la mayor parte del día sentado en una silla enfrente del televisor con el delirio estable del ordenador e infraestimando su edad en 10 años. Mantenía que tenía la misma edad, a pesar de haber pasado un cumpleaños y haber aceptado una pequeña fiesta de cumpleaños en el hospital. 


\section{Caso 2}

El Sr. B es un varón caucásico de 49 años, cuyo nacimiento y desarrollo no presentan datos clínicos relevantes. Es el segundo de dos hermanos, sin historia conocida de enfermedad psiquiátrica. Fue a una escuela especial entre los 5 y los 16 años. Dejó la escuela sin graduarse y empezó a realizar trabajos manuales durante 8 años. Es un hombre soltero que no ha tenido ninguna relación sexual de ninguna clase.

Su historial psiquiátrico empezó a la edad de 25 años, cuando comenzó a abandonarse, sintiéndose insignificante, deambulando por las calles sin dormir y, en ocasiones, sin comer. Comenzó a convertirse en una persona distante y dejó de ir a trabajar. Fue ingresado en un hospital psiquiátrico durante 5 meses, en el que fue diagnosticado de esquizofrenia. Fue tratado con antipsicóticos y, tras el alta, se le pautó tratamiento antipsicótico depot.

En este tiempo hubo una leve mejoría de su sintomatología negativa. Tuvo 6 ingresos posteriores en los siguientes 24 años. En todos los ingresos presentó sintomatología negativa. Fue tratado con clozapina durante 2 años. Sin embargo, aunque se consiguió un gran cambio en sus síntomas negativos, su familia y él no estaban contentos con los controles periódicos sanguíneos y pidieron que se suspendiera el tratamiento con clozapina.

Tras suspender dicho tratamiento comenzó de nuevo a deambular por las calles y a pararse en mitad del tráfico, por lo que el personal de la residencia en la que vivía comenzó a preocuparse. Le ingresaron de nuevo en el hospital para revisar su medicación. En el ingreso se quejó de que "se le ofuscaba el pensamiento" y se sentía débil. Daba la impresión de estar descuidado y sucio, sin contacto ocular en ningún momento. Era difícil establecer un buen entendimiento mutuo con él. Su humor era aplanado y su discurso no era espontáneo, sino circunstancial y muy pausado. Carecía de ideación suicida y de sintomatología positiva de ningún tipo. La valoración de su funcionamiento cognoscitivo reveló que estaba desorientado en el tiempo y en el espacio.

Insistía en que su edad era de 44 años. Incluso después de corregirle, insistía en que su edad era de 44 años porque su madre se lo había dicho así antes de morir (había fallecido hacía 5 años). Podía memorizar 5 ítems de nombres y direcciones, pero sólo podía evocar 2 de ellos. No podía realizar la serie de 7's pero era capaz de decir los meses del año al revés. Su nivel de información general era razonable.

Sus análisis de sangre y bioquímica eran normales y su puntación en el test de inteligencia era de 75 (era la misma que en su primer ingreso, 24 años antes). Su RM mostró un desarrollo parcialmente anormal del cuerpo calloso. Su nivel de comunicación con el personal del hospital y con otros pacientes mejoró con risperidona $(3 \mathrm{mg}$. I dos veces al día) y se le pudo reinstalar en su residencia de rehabilitación.

\section{Discusión}

Steven et al. (1978), concluyeron que la desorientación de la edad podía ser una característica de un tipo de esquizofrenia de comienzo temprano y de mal pronóstico. Aunque en el Caso 1 la edad de comienzo es aparentemente tardía, en el Caso 2 el comienzo es temprano. El pronóstico es pobre en ambos casos, debido a la sintoma- 
tología negativa con aplanamiento afectivo, conducta de retraimiento y delirios inalterables, de estar siendo controlado por un ordenador en el Caso 1, y de alteraciones orgánicas congénitas en el Caso 2. Eran ostensibles la desorientación de la edad y la falta de responsabilidad en la toma de medicación.

Todos los investigadores advierten que la edad subjetiva está relacionada con la edad al ingreso (Stevenson et al. 1978), hecho que no se da en el Caso 1 . Aunque no sucedió estando en un hospital psiquiátrico, el único incidente significativo relacionado con su edad subjetiva fue su divorcio, que ocurrió cuando él tenía 31 años; en el Caso 2 , el único evento significativo fue que su madre se lo dijo antes de morir, lo cual es un pensamiento bien establecido.

Aunque el Caso 2 se daba cuenta de que su madre había muerto 5 años antes, era inflexible con el hecho de que su edad no cambiaba. Buhrich et al. (1988), tras estudiar la desorientación de la edad en pacientes de un hospital de larga estancia diagnosticados de esquizofrenia crónica, encontraron que el deterioro cognoscitivo total se hace presente a lo largo de la enfermedad y no está relacionado con el tratamiento con neurolépticos. Esto es consistente con el Caso 1, ya que este paciente no tomaba ninguna medicación, ni fármacos médicos habituales, ni psicotropos, según la historia del médico de cabecera.

Crow y Stevens (1978), pusieron de manifiesto que puede ocurrir que la desorientación de la edad sea un delirio aislado relacionado específicamente con la noción del paciente acerca de su propia edad. En ese caso, sería esperable que la apreciación de los pacientes de otros aspectos del paso del tiempo estuviera intacta y hubiera una inconsistencia entre esta apreciación y sus respuestas a preguntas sobre su edad.
No obstante, en este contexto, sería una forma de falsa identificación de uno mismo y debería ser incluída dentro del síndrome de falsa identificación delirante. El recuerdo correcto de la propia edad depende presumiblemente de que la información sea revisada y actualizada con el paso del tiempo, es decir ¿de un proceso continuado de "teaming"? En algunos pacientes demenciados y con retraso mental es esperable que fallen acerca de su edad, pero hay poca información recogida que nos haga esperar tal carencia en pacientes psicóticos (Crow y Mitchell 1975). El reconocimiento de la edad propia es parte integral de la propia identificación.

Sin embargo, en la bibliografía no hay mención de la falsa identificación de la edad en ningún informe de casos publicados con intermetamorfosis inversa ("reverse intermetamorphosis") (Silva y Leong 1994). Podría sugerirse que la desorientación de la edad es una de estas dos opciones: una falsa identificación de la edad, como en el Caso 1 en el que la desorientación de la edad es un delirio no relacionado con una alteración cognoscitiva y, en segundo lugar, una desorientación de la edad que forma parte de un deterioro cognoscitivo.

\section{Bibliografía}

BUHRICH, N., CROW, T.J., JOHNSON, E.C., OWENS, O.G.C. Age disorientation in chronic schizophrenia is not associated with pre-morbid intellectual impairment or past physical treatment. The British Journal of Psychiatry, 152, 466-469, 1988.

CROW \& STEVENS, M. Age disorientation in chronic schizophrenia is associated with global intellectual impairment. The British Journal of Psychiatry, 133, 137-142, 1978.

CROW, T.J., MITCHELL, W.S. Subjective age in chronic schizophrenia: Evidence for a subgroup of patients 
with defective learning capacity. The British Journal of Psychiatry, 126, 360-363, 1975.

LIDDLE, P.F., CROW, T.J. Age disorientation in chronic schizophrenia is associated with global intellectual impairment. The British Journal of Psychiatry, 144, 193195. 1984.

MANSCHRECH, T.C, MAHER, B.A., WINZIGL, CANDEL, S.F., BEAUDETTES \& BOSHES, R.C. Age disorientation in schizophrenia: an indictor of progressive \& severe psychopathology, not institutional isolation. Journal of Neuro-Psychiatry and Clinical Neuro-Sciences, 12 (3), 350-358, 2000.

MOSELHY, H., OYEBODE, F. Delusional misidentification syndromes: a review of the anglophone literature. Neurology, Psychiatry and Brain Research, 5, 21-26, 1997.

SELTEN, J.P.C.J., CATH, D.C. The prevalence of age disorientation in schizophrenia patients on Dutch long stay wards. Schizophrenia Research, 11(2), 102, 1994.

SILVA, J.A., LEONG, G.B. Delusions of psychological change of self. Psychopathology 27: 285-290, 1994.
SMITH, J.M., OSWALD, W.T. Subjective age in chronic schizophrenia. The British Journal of Psychiatry, 128, 100, 1976.

STEVEN, M., CROW, T.J., BOWMAN, M.J., COLES, E.C. Age disorientation in schizophrenia: a constant prevalence of $25 \%$ in a chronic mental hospital population. The British Journal of Psychiatry, 133, 130-136, 1978.

ZANGWILL, O.L., CAUTAB, M.A. Disorientation for age. Journal of Mental Science, 99, 698-701, 1959.

Dirección para correspondencia:

Anchor Project

Sandwell Mental Health NHS Trust

2 St Michaels Court

Victoria Street

West Bromwich

B70 8ET

UNITED KINGDOM

e-mail: omaralgommer@hotmail.com 